\title{
Various Aspects of LEACH in Wireless Sensor Network: A Survey
}

\author{
Rohit koge \\ Dept. of Electronics and Communication \\ Engineering \\ Mittal Institute of Technology
}

\author{
Achint Chugh \\ Dept. of Electronics and Communication \\ Engineering \\ Mittal Institute of Technology
}

\begin{abstract}
LEACH technique is a subpart of WSN. It's an autonomous system which is connected with wireless technology. Now in the current time there is a huge demand of wireless sensor routing network devices like mobile phones, palmtop, laptop, cellular phones, PDA, s and other devices. This technology is consisting tons of very much tiny sensor nodes. This technique is able to monitor both the environment for civil and military applications. In this technique communication protocol which have significant impact of energy dissipation of these networks. Mobile adhoc network consist a group of mobile nodes which is liable to communicate with other nodes with the same infrastructure.
\end{abstract}

\section{Keyword: LEACH, Energy Efficiency, Data Aggregation, Production Cost.}

\section{INTRODUCTION}

WSN is a group of wireless nodes, which rapidly and suddenly moves, for one end to another end without any centralized controlling. Mobile adhoc network communicate with other nodes without any infrastructure. WSN allow nodes to share or exchange data with different types and characteristics from one node to another node, if nodes which belongs to sender or receiver categories, would not in transmission range of one to other, with immediate effect intermediate nodes will served as intermediate route which communicate with all the nodes of network.

The ideal wireless sensor is networked and scalable, fault tolerance, consume very little power, smart and software programmable, efficient, capable of fast data acquisition, reliable and accurate over long term, cost little to purchase and required no real maintenance. The ways how to effectively route the collected data among nodes are the utmost important topic in WSNs because of the low powered sensor nodes. Leach is the very renowned routing protocol those are using cluster based routing for the arrangement of less energy consumption. In wireless sensor routing network LEACH [3, 4] is most useful routing protocol. The cluster head receive the signals with good strength from the node then send to the sink. Conventional network protocol, like direct transformation, low transmission power, multi-hop routing, and clustering also have drawbacks which are not desirable. LEACH includes distributed cluster formation, local process to reduce global communication and randomly rotation of the cluster heads. These features allow LEACH to achieve the desired properties. WSN contains mobile nodes based on battery operated. And have very limited battery resources. Also topology of the WSN is dynamic and depends upon the movement of the nodes so accordingly it can change rapidly and unexpectedly. This changing topology affects the routing of packets which causes routing overhead, packet loss, and delay. For routing of packets and enhancing the network performance, conventional routing protocols do not work efficiently in WSN as expected therefore energy efficient routing protocols are used in the network. Work of these protocols to find out the shortest route for communicating between both the nodes. There are so many of the sensors are available in the sensor networks for the sensing process. It is capable to make these nodes cheaper and low energy efficient as much as possible and reliable to make highest quality result. The network protocol must be designed to find out the fault tolerance if there is any of the failure occurs while minimum energy consumption. In the network the sharing of the wireless sensor bandwidth must be limited, and should be capable to perform local collaboration to reduce bandwidth requirements. There are some of the models for the micro sensor networks.

- The location should be fixed and distance must be far from the sensors.

- All nodes should be same configured, and energy constrained.

WSNs offer unique benefits and versatility in terms of lowpower and low-cost rapid deployment for applications which do not require human supervision. Routing strategies and security issues are a great research challenge now days in WSN but in this paper we will emphasize on the network routing protocol. The main work of WSN is to sense and select the data from target nodes, after that process on that data, also sending data to the target node directly. But this task would not be feasible as the required transmission energy increases proportionally to the square of the distance. Therefore, data is routed using multi-hop communication. Achieving this task efficiently requires the development of an 


\section{INTERNATIONAL JOURNAL ONLINE OF SCIENCE}

\section{ISSN: 2455-0108}

energy efficient routing protocol to set up paths between sensor nodes and the data sink. As several alternative routes to a destination node may exist, the routing decision has a significant effect on load balancing, end-to-end reliability and latency. The path of the network should be short for the network life. Due to resource constraints, WSN poses considerable challenges ranging through network organization, topology discovery, communication scheduling, routing control and signal processing. WSNs are not centralized one as no static infrastructure exists. Peer-to-peer communication exists between nodes. Multi-hopping can cause a sensor node to communicate with a node that is not in radio range of each other via intermediate nodes.

The WSNs must be flexible make addition and removing nodes very easily. Also network should be able to divided into no. of clusters called clustering, in this network one sensor node is get choose as head cluster and remaining all nodes are treated as cluster members. All nodes work with each other with the services and request, the head cluster collect the data from the each cluster members, after the collection of data, data aggregation is done by cluster head to remove data redundancy then forward to the sink, the cluster head consume much energy as compare to cluster members. The workload of the cluster heads is get distributed by the rotation of the nodes with comparing of the energy consumption is called cluster head.

\section{APPliCATIONS}

There are some of the applications of LEACH

\section{a) Data delivery model:}

Data delivery model overcomes the problem of fault tolerance domain by providing the alternative path to save its data packets from nodes or link failures [13]. It seriously the routing protocol in WSN, especially with regard to use the limited energy of the node, for security purpose [14], energy consumption and route immobility.

\section{b) Scalability:}

The system which said for scale if it is effectiveness increases Whenever any of the hardware is adding and also proportional To the capacity added [15].

\section{c) Resilience:}

Sometimes, due to environment problem or battery consumption sensors erratically stop working [16]. This problem is overcome by finding the alternate path when current-in use nodes stop operating.

\section{d) Production cost:}

As it is very much clear that one need to make low cost to the network and to maintain this low cost one need to keep the least cost of the sensor node. e) Data aggression/fusion:

To enhance the life time of the network, technique of data aggregation come into the picture. This technique of data aggregation is responsible to for doing so by the means of various aspects i.e. min, suppression, average and max and this is to get energy efficient along with traffic optimization in various network routing protocols [18].

\section{ENERGY EFFICIENCY WITH LEACH}

Cluster heads are main relying sensor nodes as these are taking the duty of data communication to the base station, dissipate more energy. The operation of LEACH head and LEACH head-C is controlled by rounds, which consist of two stages setup stage and steady state stage. Cluster heads are selected in setup phase and allocate the TDMA schedule to the respective CMs. While in the steady state phase, data communication between the CMs and the cluster head is performed. A CM in a cluster is active only during its allocated time slot, while cluster heads are active all the time in steady state phase. LEACH head performs periodic cluster head selection; the energy utilization burden of the cluster heads is also shared. The duration of the steady state phase is longer than the duration of the setup phase. Study shows that LEACH head provides a factor of 4-8 reduction in energy consumption compared to a flat architecture routing protocol. Major disadvantage of this protocol is that they do not consider the residual energy of sensor nodes and assume zero energy consumption for the formation of cluster. In LEACH-E protocol, initially all nodes have same energy and same probability of becoming the cluster head. After the first round, energy level of each node changes. Then the amount of residual energy of each node is used to select cluster head nodes. The nodes with highest residual energy are preferred on rest of the nodes. LEACH-E enhance lifetime of network by balancing energy load including all the nodes present in the network [11] [12].The LEACH is based on a hierarchical clustering structure model and Network routing protocol for network based on energy efficient clusters. This model use an local cluster head in the local network and also with the same process for the another network which is making another local cluster heads, then sender send the information or data to the local heads the heads sent the same information or the data to another heads, other head receive the data, there after cluster head send data to the receiving end nodes, this process will take least energy for the process.

LEACH is the hierarchical routing protocol that use cluster based routing in order to minimize the energy consumption and further increase the network performance in WSN. It consists of many mobile nodes, communicating with each other in the network. These mobile nodes are powered by battery. The resource constrained nature of WSN suffer from many challenges in its design and operation, which degrades its performance and also the major fact that mobile nodes run 


\section{IJO-SCIENCE}

\section{INTERNATIONAL JOURNAL ONLINE OF SCIENCE}

\section{ISSN: 2455-0108}

out of energy quickly, This will quickly drain the battery of the nodes and reduce the system lifetime. However, the only receptions in this protocol occur at the base station, so if either the base station is close to the nodes, or the energy required to receive data is large, this may be an acceptable (and possibly optimal) method of communication.

LEACH operates in several rounds, each consisting of a set-up and a steady-state phase. Each node transmits sensed data to its closest $\mathrm{CH}$. The $\mathrm{CH}$ for each cluster receives and aggregates the data from cluster members and then transmits the aggregated data to the BS through a single-hop relay. LEACH creates a set-up phase for $\mathrm{CHs}$ ' selection, and a steady-state phase for time slot scheduling and transmission. WSNs which consists lots of small battery-powered devices are frequently to obtain various sorts of useful data from surroundings. Advanced nodes create a source of heterogeneity in the network and these nodes would have higher level of energy as compare to normal nodes. Cluster Head could be elected by the means of weighted election probabilities which is of every node and this is based on residual energy level of various nodes. Threshold for normal and advanced nodes are calculated by using weighted probabilities.

The minimum amplifier energy of a transmitter or receiver is directly proportional to the square of the distance [19]. Thus long distance transmission increases the energy consumption in a WSN. Communication energy of WSNs is much more than sensing and computation energy.

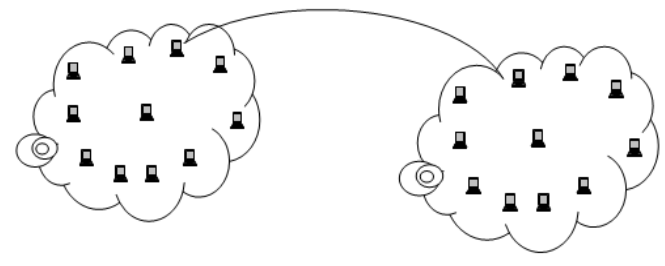

Figure 1 Low-Energy Adaptive Clustering Hierarchy

\section{ADVANTAGES AND DISADVANTAGES OF LEACH}

\section{Advantage of LEACH}

1. The Cluster Heads aggregates the whole data which lead to reduce the traffic in the entire network.

2. As there is a single hop routing from nodes to cluster head it results in saving energy.
3. It increases the lifetime of the sensor network

4. In this, location information of the nodes to create the cluster is not required.

5. LEACH is completely distributed as it does not need any control information from the base station as well as no global knowledge of the network is required.

Disadvantage of LEACH

1. LEACH does not give any idea about the number of cluster heads in the network

2. One of the biggest disadvantage of LEACH is that when due to any reason Cluster head dies, the cluster will become useless because the data gathered by the cluster nodes would never reach its destination i.e. Base Station.

3. Clusters are divided randomly, which results in uneven distribution of Clusters. For e.g. some clusters have more nodes and some have lesser nodes. Some cluster heads at the center of the cluster and some cluster heads may be in the edge of the cluster; this phenomenon can cause an increase in energy consumption and have great impact on the performance of the entire network

\section{LITERATURE REVIEW}

Energy efficient routing protocols in WSN and classified them according to the get approaches employed to all of them for low energy consumption. The transmission control should be employed when the communication between the nodes take place at regular intervals whereas the load distribution approach is used where the node density or the traffic density is not uniform and hence need is to employ the equal distribution of load to low energy consumption. The Sleep/Power down approach should be used where the communication between the nodes is low and hence, they can utilize their energy when there is energy imbalance problem. Since energy is a constrained resource in WSN, and also more research is to be done to find energy efficient protocols. [5]

To solve this problem, they proposed a new approach to selecting cluster heads by assuming these parameters:

- The network satisfies the pre-conditions of applying LEACH protocol.

- When the setup has been done then, through GPS node are able to know there position

- The transmission of power is adjustable in the all nodes. If necessary they can communicate with the base stations to acquire the initial setting information of the network. 


\section{INTERNATIONAL JOURNAL ONLINE OF SCIENCE}

\section{ISSN: 2455-0108}

The cluster heads in all other regions will be generated in the same way. The cluster heads generated with this approach will not be close to each other.

In new version of $\mathrm{LEACH}$ protocol, the cluster contains; $\mathrm{CH}$ (responsible only for sending data that is received from the cluster members to the $\mathrm{BS}$ ), vice-CH (the node that will become a $\mathrm{CH}$ of the cluster in case of $\mathrm{CH}$ dies), cluster nodes (gathering data from environment and send it to the $\mathrm{CH}$ ). In $\mathrm{V}$-LEACH protocol, besides having a $\mathrm{CH}$ in the cluster, there is a vice- $\mathrm{CH}$ that takes the role of the $\mathrm{CH}$ when the $\mathrm{CH}$ dies.

The cluster set up phase is divided into two sub clustering phases. In first sub clustering phase, the base station has to cluster the sensor nodes and assign the proper roles to them. This operation is referred as MAP protocol. In second sub clustering phase, only the overlapping clusters are newly formed, in which the normal node may get changed to cluster head node or the cluster head node may get changed to cluster node. This phase is referred as REDUCE protocol.

As the result, the network lost its service ability, despite of a large amount of residual energy of the other nodes. A sensor in WSNs periodically produces data as it monitors its area. The fundamental operation in such a network is the systematic gathering (with or without innetwork aggregation) and transmitting of sensed data to a base station for further processing.

A key major challenge in WSNs is to schedule nodes' activities to reduce energy consumption. This research work focused on deceitful energy-efficient protocols for low data rates WSNs, where sensors consume lot of energy in different radio states (sleeping, listening, receiving, transmitting, and keep idle) and consume energy for state transition.

It is a group of sensor nodes, and these nodes are positioned at locations where their energies are less than a threshold. The results and study show that planned FZ-LEACH algorithm outperforms LEACH in terms of energy consumption also with the network existence.

A node in network is no longer useful when its battery dies so we use LEACH. It space out the lifespan of the nodes allowing it to do the only minimum work it needs to transmit data. It has 2 phases: setup phase, where cluster head are chosen and steady phase, in which $\mathrm{CH}$ is maintained when data is transmitted between nodes. Goal of LEACH is to increase the life of network. It is clustering based routing protocol minimizes global energy usage by distributing load to all nodes at different point in time.

DSDV, AODV, DSR for better network performance in WSN without much packet loss using simulation on NS2 based on parameters mobility, load and size of the ad hoc network.

Design issues of routing protocols consists of fault tolerance, scalability, production costs, operating environment, power consumption, data delivery models, data aggregation/fusion, quality of service, data latency and overhead, node deployment and classification of protocol based on flat, hierarchical, data centric, location centric, power usages, data aggregation, scalability, overhead, data delivery model, and quality of service. [7] In the Set-up phase the main goal is to make cluster and select the cluster head for each of the cluster by choosing the sensor node with maximum energy. Steady Phase which is comparatively longer in duration than the setup deals mainly with the aggregation of Data at the cluster heads and transmission of aggregated Data to the Base station.

LEACH consists many number of clusters members along with cluster heads. So the responsibilities of the cluster head is to collect data from the cluster nodes, then compare and transmit the data to the base station, all nodes provide in the slotted tie to respond in the order, so the LEACH use the radio model to stop energy consumption by the transmission process.

\section{CONCLUSION}

The LEACH protocol is designed for making of low use of the energy which is very useful network life or use in the emergency areas. WSNs keeps thousands of very small, cheap cost less power and multifunctional sensor nodes, which are having very low battery life, many of the energy efficient algorithms designed for this problem. The field of WSNs is very challenging and recent. Various static methods and parameters can improve the performance of the network. Research can be done on choice of cluster head. Cluster head can be chosen based on the density of nodes. Distributing the energy among the nodes in the network is effective in reducing energy dissipation from a global perspective and enhancing system lifetime. In this paper discussed about LEACH applications, energy efficiency in leach, advantage and disadvantage.

\section{References}

[1] Seapahn Megerian and Miodrag Potkonjak, "Wireless sensor networks," Book Chapter in Wiley Encyclopedia of Telecommunications, Editor: John G. Proakis, 2002.

[2] Heinzelman W., Chandrakasan A., and Balakrishnan H., "Energy Efficient Communication Protocol for Wireless Microsensor Networks".2000.

[3]GuofengHou, K. Wendy Tang "Evaluation of LEACH Protocol Subject to Different Traffic Models" The $1^{\text {st }}$ International Conference on Next Generation Network (NGNCON 2006) Hyatt Regency Jeju, Korea / July 9 - 13, 2006, IEEE

[4] W. Heinzelman, A Chandrakasan, and H Balakrishnan, "Energy Efficient Communication Protocols For Wireless Microsensor 


\section{IJO-SCIENCE}

\section{INTERNATIONAL JOURNAL ONLINE OF SCIENCE}

\section{ISSN: 2455-0108}

Networks," Proc. Hawaiian International Conference on Systems Science, Jan. 2000.

[5] Swapnil Singh, Sanjoy Das, "Survey on Energy Efficient Routing Protocols in Mobile Ad Hoc Networks", International Journal of Computer, Information, Systems and Control Engineering Vol:8 No: 2, 2014

[6] Katiyar, V., Chand, N., Gautam, G.C., Kumar, A., Improvement in LEACH protocol for large-scale Wireless sensor networks, Emerging Trends in Electrical and Computer Technology (ICETECT), 2011 International Conference on , vol., no., pp.1070, 1075, 23-24 March 2011.

[7]R.V. Biradar, V. C. Patil, Dr. S. Sawant, and Dr. R. R. Mudholkar," Classification and comparison of routing protocols in wireless sensor networks", Ubi CC Journal, Vol.4.

[8] M. Bani Yassein, A. Al-zou'bi, Y. Khamayseh, W. Mardini "Improvement on LEACH Protocol of Wireless Sensor Network (VLEACH)", International Journal of Digital Content Technology and its Applications Volume 3, Number 2, June 2009.

[9] M. J. Handy, M. Haase, D. Timmermann, "Low Energy Adaptive Clustering Hierarchy With Deterministic Cluster Head Selection", IEEE MWCN, 2002.

[10] W. B. Heinzelman, A. Chandrakasan and H. Balakrishnan, "Energy-Efficient Communication Protocol for Wireless Micro sensor Networks," Proc. $33^{\text {rd }}$ Hawaii Int'l. Conf. Sys. Sci., Jan. 2000.

[11] M.Ahmad Jan and M.Khan, "A Survey of Cluster based Hierarchical Routing Protocols," IRACST International Journal of Computer Networks and Wireless Communications (IJCNWC),vol.3, no.2, pp. 138-143, April 2013.

[12] M.Usha and Dr.N.Sankarram, "A Survey on Energy Efficient Hierarchical (Leach) Clustering Algorithms in Wireless Sensor Network," International Journal of Innovative Research in Computer and Communication Engineering (IJIRCCE), Proceedings of International Conference On Global Innovations In Computing Technology (ICGICT'14), vol.2, special issue 1, pp. 601- 609, March 2014.

[13] Jamal, N.Al-Karaki and Ahmed E. Kamal, "Routing techniques in wireless sensor networks: a survey", Wireless Communications, IEEE, vol. 11, pp. 6-28, Dec. 2004.

[14] Jim Farmer, "Managing Secure Data Delivery: A Data Roundhouse Model", August. 2001.

[15] S. Muthukarpagam, V. Niveditta and Nedunchelivan, "Design issues, Topology issues, Quality of Service Support for Wireless Sensor Networks: Survey and Research Challenges", IJCA Journal, (2010).

[16] Luis Javier GarcíaVillalba and Ana Lucila Sandoval Orozco, "Routing Protocols in Wireless Sensor Networks", October 2009.

[17] Sidra Aslam, Farrah Farooq and ShahzadSarwar, "Power Consumption in Wireless Sensor Networks", Proceedings of the 7th International Conference on Frontiers of Information Technology, (2009).

[18] Nandini .S.Patil and P. R. Patil, "Data Aggregation in Wireless Sensor Network", IEEE International Conference on Computational Intelligence and Computing Research, 2010.

[19] W. Heinzelman,A.Chandrakasan , and H. Balakrishnan , "An application specific protocol architecture for wireless microsensor networks," IEEE Transactions on Wireless Communications, vol. 1, no. 4 , pp. 660 - 670, Oct. 2002. 